\title{
Tuning Rheological Performance of Silica Concentrated Shear Thickening Fluid by Using Graphene Oxide
}

\author{
Wenchao Huang, ${ }^{1}$ Yanzhe Wu, ${ }^{1}$ Ling Qiu, ${ }^{1}$ Cunku Dong, ${ }^{1}$ Jie Ding, ${ }^{2}$ and Dan Li ${ }^{1}$ \\ ${ }^{1}$ Department of Materials Engineering, Monash University, Wellington Road, Clayton, VIC 3800, Australia \\ ${ }^{2}$ Defence Science and Technology Organisation, Land Division, 506 Lorimer Street, Fishermans Bend, VIC 3207, Australia \\ Correspondence should be addressed to Jie Ding; jie.ding@dsto.defence.gov.au
}

Received 9 December 2014; Revised 26 February 2015; Accepted 26 February 2015

Academic Editor: Victor V. Moshchalkov

Copyright (c) 2015 Wenchao Huang et al. This is an open access article distributed under the Creative Commons Attribution License, which permits unrestricted use, distribution, and reproduction in any medium, provided the original work is properly cited.

The addition of a small amount of graphene oxide into a traditional colloidal silica-based shear thickening fluid (STF) can lead to a significant change in viscosity, critical shear rate, storage modulus, and loss modulus of STF. This finding provides an effective way to prepare stronger and light-weight STFs.

\section{Introduction}

Graphene oxide (GO) is a graphene sheet functionalized with various oxygen-containing functional groups, which inherits many merits from graphene, including exceptionally high mechanical strength, and high aspect ratio/specific surface area but it can be well dispersed in some solvents including water [1-3]. GO has attracted tremendous interest in the last few years, because of not only its use as a precursor for graphene but also its unique colloidal behaviours, such as amphiphilic property, liquid crystal, and viscoelastic behaviour $[1,4-8]$. In particular, the unique viscoelastic behavior of GO dispersion is fundamentally different from traditional viscoelastic materials such as polymers, wherein $\mathrm{GO}$ is able to form a gel at very low concentrations as the outstanding reinforcing effect is ascribed to a mechanical percolation phenomenon $[2,9-11]$. Understanding the viscoelastic behaviour of GO is both of theoretical and practical importance in synthesis of graphene-based materials, including graphene fibers/foams and its composite materials [12-16]. Despite the significant work that has been done on exploring the colloidal behaviour of GO itself [11], the understanding on rheological behaviour of GO in complex colloidal systems remains largely unexplored.

Shear thickening is one of the most interesting rheological phenomena of colloidal suspensions observed over the past decades [17-20]. Shear thickening, by definition, is a dramatic increase in viscosity when the shear rate of certain mechanical deformation/perturbation increases to a critical value. This unique feature enables the use of STFs in body armours and energy absorbers, as it can provide advantages of light-weight and flexibility compared with conventional fibre-based body armours [21]. The shear thickening phenomenon has previously been observed in various fluids or suspensions and several mechanisms have been formulated involving the order-disorder transition [22, 23], impact activated solidification [24], and hydrodynamic formation of clusters [19, 25-27]. For the reversible shear thickening of colloidal suspensions, the widely accepted mechanism is the shear-induced hydroclusters transitionally sized together by hydrolubrication forces [27, 28]. As shown in Figures S1(a) and (b) in Supplementary Material available online at http://dx.doi.org/10.1155/2015/734250, shear thickening behaviour is caused by the formation of a clustering structure at the higher strain rate, hindering the movement of free particles through it $[18,27]$. At present, the most studied STF is based on the suspension of silica in polyethylene glycol (PEG) matrix. The hydrophilic fumed silica nanoparticles are stably dispersed in PEG through hydrogen bonding to obtain the silica concentrated STF [28]. It is noteworthy that the shear thickening behaviour is strongly dependent on the particle size and volume fraction [27]. In this work, we explore 
how the introduction of two-dimensional nanoparticle, $\mathrm{GO}$ sheets, can change the rheological properties of the silicabased STF.

\section{Materials and Methods}

2.1. Materials. Fumed silica nanoparticles (S5505) and polyethylene glycol (PEG-200) were purchased from SigmaAldrich Company. The fumed silica particles were hydrophilic, containing the hydroxyl groups on the surface with crystal size of $14 \mathrm{~nm}$ in diameter. The surface area of these nanoparticles is $200 \mathrm{~m}^{2} / \mathrm{g} \pm 25 \mathrm{~m}^{2} / \mathrm{g}$. The silica primary particles were irreversibly fused into large aggregated structures that cannot be disrupted by shear. GO dispersions were synthesized by using a similar procedure, reported by our group previously $[1,7]$. Before the experiments, the GO was ultrasonicated for 10 mins to achieve a uniform dispersion.

2.2. Preparation of STFs. Silica/PEG samples were prepared by adding two different weight percentages of silica (15\% and $20 \%$ ) into PEG-200 and a combination of manual mixing and ultrasonication processes were used. The mixture was stirred manually for 10 minutes firstly and then put in the ultrasonicator for 3 hours until a homogenous mixture was achieved. Silica/GO/PEG samples were prepared by adding different concentrations (weight concentration) of the exfoliated GO to the prepared silica/PEG. The mixture of GO and silica concentrated STF experienced a second dispersion cycle by ultrasonication. After proper mixing, the excess water was removed via an evaporation process by heating up the samples in the oven at $80^{\circ} \mathrm{C}$ overnight. It was found that the suspensions showed no visible evidence of phase separation, precipitation, or increases in turbidity over long periods of time (four months).

2.3. Characterization. The rheometer (ARES) was employed to determine the rheological performance of silica-based suspension. Both steady shear and dynamic shear methods were carried out at the room temperature. The steady test was conducted by cone-and-plate geometry with $0.02 \mathrm{rad}$ in angle and $25 \mathrm{~mm}$ in diameter and the dynamic test was performed by two parallel plates with $25 \mathrm{~mm}$ in diameter and $500 \mathrm{~mm}$ in gap. All the samples were presheared at shear rate of $1 \mathrm{~S}^{-1}$ for $180 \mathrm{~s}$ and then were relaxed for $300 \mathrm{~s}$ before both steady and dynamic tests. A preshear process is necessary for achieving a better dispersion.

Scanning electron microscope images were captured by JEOL 7001. The samples prepared for SEM were spun from the dilute STF (with and without GO). All samples were deposited on the high conductive silicon wafer.

\section{Results and Discussion}

The silica-based STF was prepared by dispersing the fumed silica in PEG followed by mechanical stirring. GO was preexfoliated in an aqueous solution with the same method reported in our previous paper [29], followed by mixing with the silica-based STF. After mixing, the water was removed by

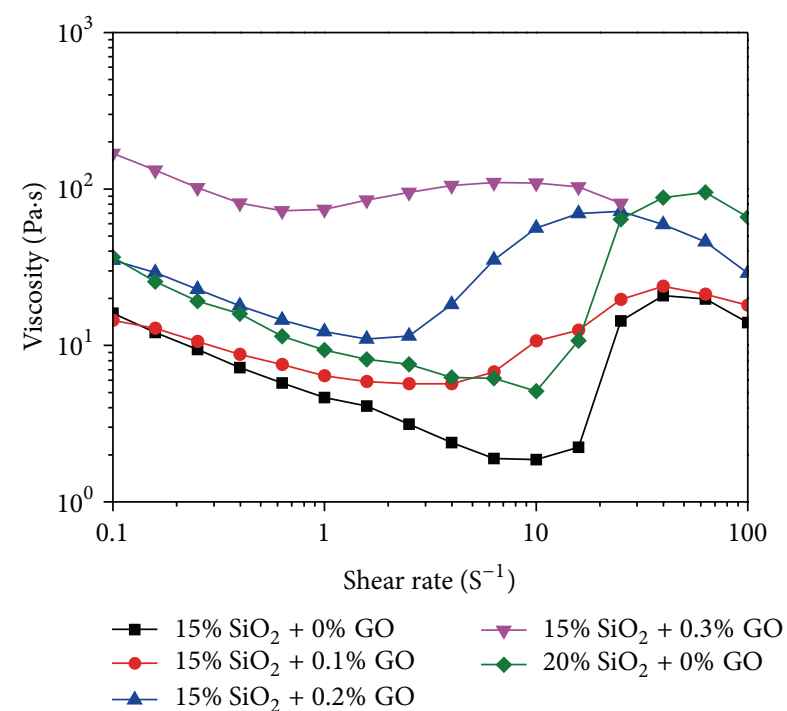

FIgUre 1: Steady shear sweep test of silica/PEG-based STF with various loading amounts of $\mathrm{GO}$ and silica (the numbers in the inset represent the concentration of each component in weight ratio).

evaporation and GO sheets were found to be well dispersed in the PEG matrix. The prepared suspension is rather stable under the ambient conditions and no significant colour change and aggregation of GO were observed over four months.

Figure 1 presents the steady shear test results. The silicabased STF follows the typical shear thickening behaviour, experiencing a slight decrease of viscosity with shear rate at the low shear rate region, followed by a dramatic increase of viscosity when the shear rate is increased to a certain point or so-called critical shear rate, after which the viscosity is decreased with the shear rate. The rheological behaviours of STF strongly are dependent on the particle shape, concentration, and the interaction between these elements; hence tuning the silica content is a conventional way to change the rheological behaviour of STF $[17,28]$. Figure 1 also illustrates the effect of an increasing silica fraction on the shear rate dependence of viscosity for the silica-based STFs. At the beginning of the steady shear test, the viscosity increases from $16 \mathrm{~Pa} \cdot \mathrm{s}$ in $15 \%(\mathrm{w} / \mathrm{w})$ silica sample to $35 \mathrm{~Pa} \cdot \mathrm{s}$ in $20 \%$ $(\mathrm{w} / \mathrm{w})$ silica sample. In addition, at $15 \%(\mathrm{w} / \mathrm{w})$ silica in PEG, a continuous shear thickening region appears at the higher critical shear rates of $16 \mathrm{~S}^{-1}$. When the silica fraction is increased to $20 \%(\mathrm{w} / \mathrm{w})$, the critical shear rate shifts to $10 \mathrm{~S}^{-1}$. As the fraction of particles is increased, this shear thickening region progressively shifts to lower shear rates and the increase in viscosity becomes more dramatic. These trends have been observed to be consistent in a range of suspensions and a high concentration of particles is seen as a requirement observation of shear thickening at achievable shear rates [30, 31].

Compared with the silica-only STF, the introduction of GO exhibits a similar viscosity trend as a function of shear rate, but it has led to remarkable changes in the viscosity and critical shear rate with significantly lower concentrations. 


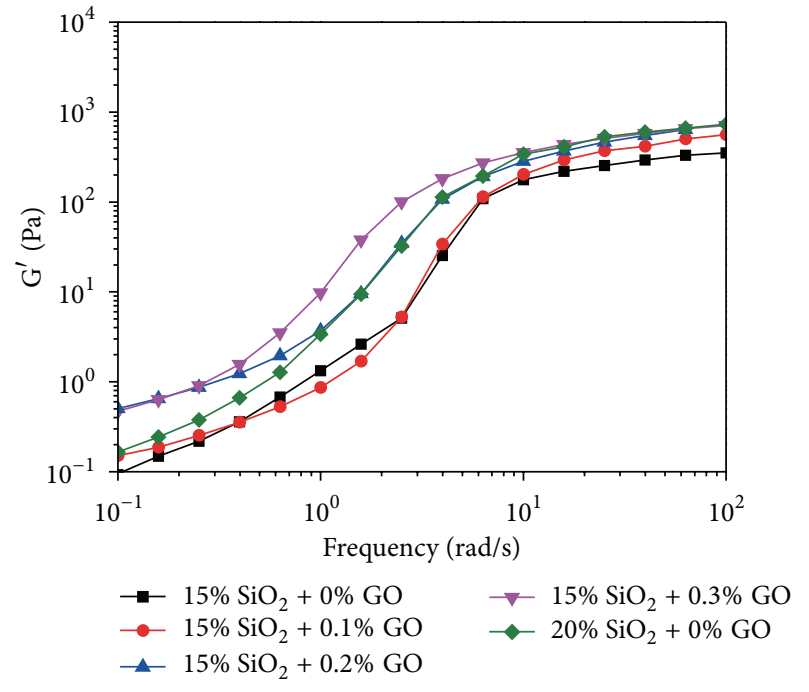

(a)

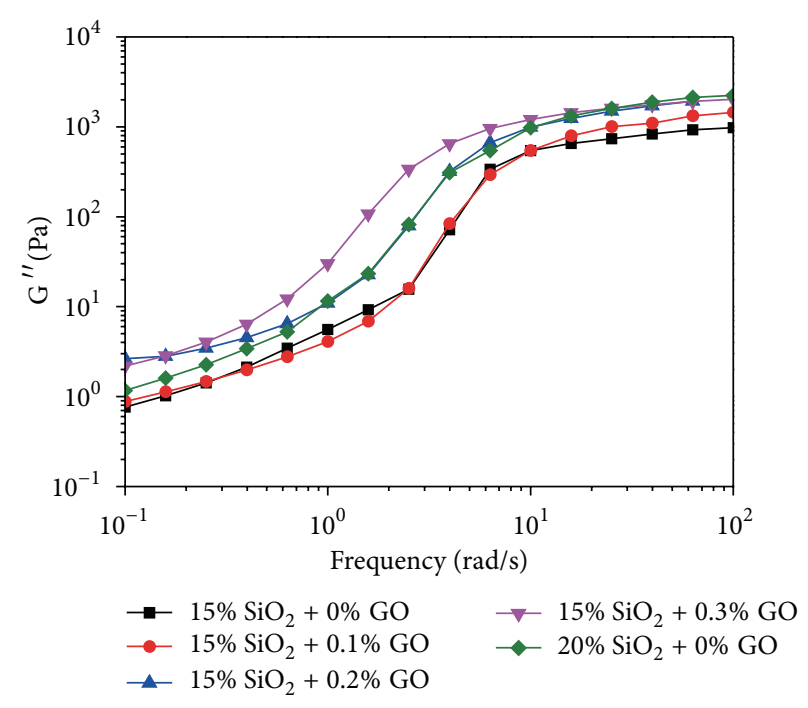

(b)

FIGURE 2: Dynamic frequency test at the constant strain of $250 \%$ showing the storage and loss modulus change as a function of frequency sweep for colloidal suspension of fumed silica in PEG with various loading amount of GO and silica (the numbers represent the concentration of each component in weight ratio). (a) Storage modulus as a function of frequency; (b) loss modulus as a function of frequency.

As the GO concentration increased from $0 \%$ to $0.3 \%$, the viscosity of the fluid increases over one order of magnitude from $16 \mathrm{~Pa} \cdot \mathrm{s}$ to $168 \mathrm{~Pa} \cdot \mathrm{s}$ (for the sample containing $15 \%(\mathrm{w} / \mathrm{w})$ silica). In addition, the critical shear rate, one of the most important parameters in STF, also exhibits a dramatic shift towards the lower critical shear rate in the fluid incorporated with GO. The critical shear rate of $15 \%$ (w/w) silica in PEG is around $16 \mathrm{~S}^{-1}$. In comparison, the corresponding critical shear rates of $15 \%(\mathrm{w} / \mathrm{w})$ silica in PEG with the additional $0.1 \%(\mathrm{w} / \mathrm{w}), 0.2 \%(\mathrm{w} / \mathrm{w})$, and $0.3 \%(\mathrm{w} / \mathrm{w})$ GO were $3 \mathrm{~S}^{-1}$, $2.5 \mathrm{~S}^{-1}$, and $1 \mathrm{~S}^{-1}$, respectively. The critical shear rate is the onset of the viscosity increase, indicating the formation of a hydrocluster structure which hinders the free particles passing through it. Apparently, the increasing GO content results in the formation of a hydrocluster structure at a lower shear rate.

We have also investigated the dynamic shear behaviour and the results are presented in Figure 2. Both the storage and loss modulus of the STFs are tested as a function of frequency. Comparing the fluid containing only $15 \%(w / w)$ silica in PEG with the samples incorporated by small amounts of GO, both the storage and loss moduli are increased over the whole frequency range tested. Particularly, the moduli of the STF with $0.3 \%(\mathrm{w} / \mathrm{w}) \mathrm{GO}$ are increased by nearly an order of magnitude. The use of GO in the fluid also shifts the onset of shear thickening behaviour towards the lower frequency compared with the bare fluid of $15 \%(\mathrm{w} / \mathrm{w})$ silica in PEG. The rapid increase of modulus at the lower frequency can be viewed as being equivalent to the rapid viscosity increase at the critical shear rate under the steady shear sweep according to the equation of Cox-Merz [32].

Both the steady and dynamic rheological tests have indicated that by adding only $0.2 \%$ GO into the STF containing $15 \%$ of silica, the viscosity, storage, and loss modulus of the fluid can be greatly enhanced to be close to that of the fluid containing $5 \%$ more silica (i.e., $20 \%$ silica in total). This finding suggests that at least $5 \%(\mathrm{w} / \mathrm{w}$, calculated from the difference between $15 \%$ and $20 \%$ ) of silica particles can be replaced by a very small amount of GO $(0.2 \%)$ to achieve a similar rheological performance. This is remarkable as this will lead to the reduction of total weight of the STF, which is favourable to many applications such as body armours.

To further investigate the role of GO in the STFs, we have also examined the viscoelastic behaviour of pure GO dispersions. Our experiment showed that the GO dispersion itself is a shear-thinning fluid (Figure S3), which is in agreement with the literature [33-35]. Thus, it is unlikely that the remarkable change of the rheological behaviour of GO-added STFs is caused by the interaction between GO sheets themselves. The interaction between GO sheets and silica nanoparticles and particularly the large aspect ratio of GO sheets could account for the significant change of the STFs' viscoelastic behaviour. GO sheets have a much larger hydrodynamic diameter than granular silica nanoparticles. Therefore, the addition of GO could cause a much more prominent congestion effect, making the hydroclusters form at the lower shear rate. Our experiments also appear to suggest that there are fairly strong interactions between silica nanoparticles and GO sheets. For example, when the mixed fluid is dried, the silica particles near the GO tend to be attracted to the GO sheets (Figures 3(b) and S2). SEM imagines (Figures 3 and S2) of the silica and GO composite also show the high aspect ratio of GO formed a barrier that could benefit the aggregation effect of dispersed silica particles. For the application of shear thickening fluid for the dissipation of mechanical impact, one has to note that it is not only the ability to absorb mechanical energy but more relevant the capability to effectively dissipate the mechanical 


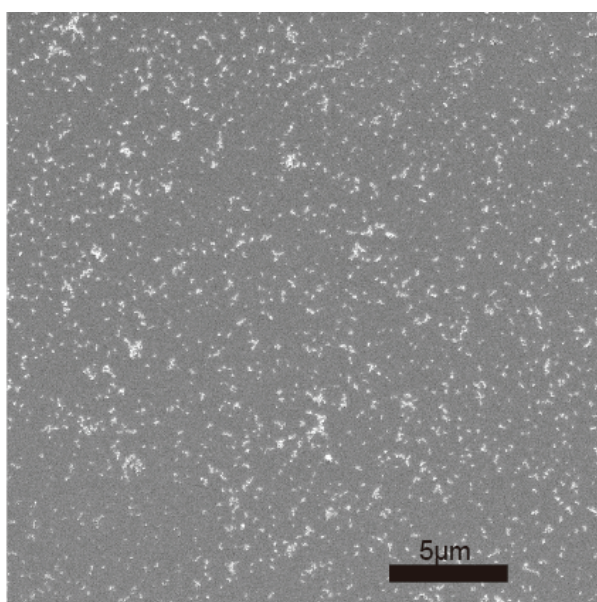

(a)

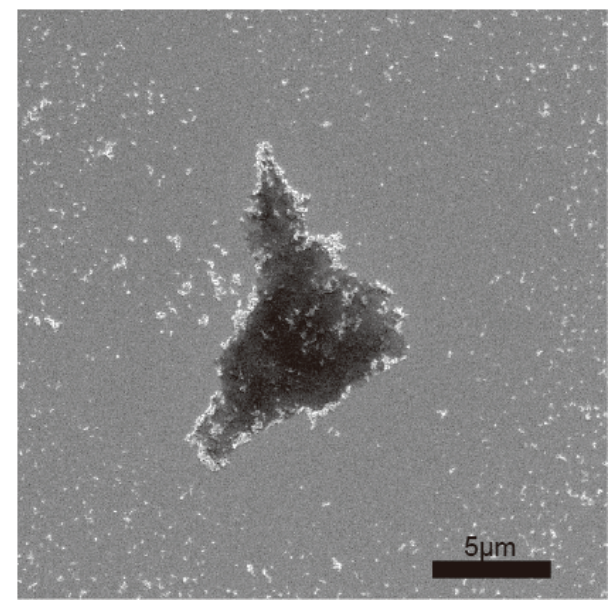

(b)

FIGURE 3: SEM images of spin-casted STFs on Si wafer: (a) 15\% silica + 0\% GO and (b) $15 \%$ silica $+0.2 \%$ GO.

peak power as well; mathematically representation is the product of viscosity times the square of shear rate for the shear thickening fluid and eventually depends on the elastic modulus and strength of transitionally formed hydrocluster structure. Therefore the effects of graphene oxide on the shear thickening fluid is envisaged to be twofold; one is the ability to initiate the shear thickening at a reduced overall weight; the other is similar to that of filler in the polymer composite, the capability to strengthen the transitionally formed hydrocluster structure in order to keep the materials structural integrity after several impacts.

\section{Conclusions}

In summary, the addition of a small amount of GO into silica/PEG-based STFs can lead to remarkable increase of the viscosity and shift of the shear thickening onset towards lower shear rate. It can also enhance the materials' storage modulus. Our finding suggests that $\mathrm{GO}$ can be utilized as a very efficient nanofiller to modify silica-based STF and provides a new route to design and fabrication of light-weight and highstrength energy absorbers. It also opens a new route to tune the onset of shear thickening to preferred regimes for certain applications. However, further experimental and theoretical study will be required to better understand and interpret the influence mechanism of $\mathrm{GO}$ on the rheological properties of the relevant silica suspensions.

\section{Conflict of Interests}

The authors declare that there is no conflict of interests regarding the publication of this paper.

\section{Acknowledgment}

This research was supported by DSTO Fellowship Program from Defence Science and Technology Organisation, Australia.

\section{References}

[1] D. R. Dreyer, S. Park, C. W. Bielawski, and R. S. Ruoff, "The chemistry of graphene oxide," Chemical Society Reviews, vol. 39, no. 1, pp. 228-240, 2010.

[2] C. Li and G. Shi, "Functional gels based on chemically modified graphenes," Advanced Materials, vol. 26, no. 24, pp. 3992-4012, 2014.

[3] D. Chen, H. Feng, and J. Li, "Graphene oxide: preparation, functionalization, and electrochemical applications," Chemical Reviews, vol. 112, no. 11, pp. 6027-6053, 2012.

[4] C. Cheng and D. Li, "Solvated graphenes: an emerging class of functional soft materials," Advanced Materials, vol. 25, no. 1, pp. 13-30, 2013.

[5] J. Kim, L. J. Cote, and J. Huang, "Two dimensional soft material: new faces of graphene oxide," Accounts of Chemical Research, vol. 45, no. 8, pp. 1356-1364, 2012.

[6] F. Bei, X. Hou, S. L. Y. Chang, G. P. Simon, and D. Li, "Interfacing colloidal graphene oxide sheets with gold nanoparticles," Chemistry, vol. 17, no. 21, pp. 5958-5964, 2011.

[7] L. Qiu, X. Yang, X. Gou et al., "Dispersing carbon nanotubes with graphene oxide in water and synergistic effects between graphene derivatives," Chemistry - A European Journal, vol. 16, no. 35, pp. 10653-10658, 2010.

[8] J. E. Kim, T. H. Han, S. H. Lee et al., "Graphene oxide liquid crystals," Angewandte Chemie International Edition, vol. 50, no. 13, pp. 3043-3047, 2011.

[9] H. Bai, C. Li, X. Wang, and G. Shi, "On the gelation of graphene oxide," Journal of Physical Chemistry C, vol. 115, no. 13, pp. 55455551, 2011.

[10] C. Li and G. Shi, "Three-dimensional graphene architectures," Nanoscale, vol. 4, no. 18, pp. 5549-5563, 2012.

[11] S. Naficy, R. Jalili, S. H. Aboutalebi et al., "Graphene oxide dispersions: tuning rheology to enable fabrication," Materials Horizons, vol. 1, no. 3, pp. 326-331, 2014.

[12] X. Li and G. B. McKenna, "Considering viscoelastic micromechanics for the reinforcement of graphene polymer nanocomposites," ACS Macro Letters, vol. 1, no. 3, pp. 388-391, 2012.

[13] R. Jalili, S. H. Aboutalebi, D. Esrafilzadeh et al., "Scalable onestep wet-spinning of graphene fibers and yarns from liquid 
crystalline dispersions of graphene oxide: towards multifunctional textiles," Advanced Functional Materials, vol. 23, no. 43, pp. 5345-5354, 2013.

[14] Y. Tan, Y. Song, and Q. Zheng, "Hydrogen bonding-driven rheological modulation of chemically reduced graphene oxide/poly(vinyl alcohol) suspensions and its application in electrospinning," Nanoscale, vol. 4, no. 22, pp. 6997-7005, 2012.

[15] L. Chen, Y. He, S. Chai, H. Qiang, F. Chen, and Q. Fu, “Toward high performance graphene fibers," Nanoscale, vol. 5, no. 13, pp. 5809-5815, 2013.

[16] L. Wu, B. Zhang, H. Lu, and C.-Y. Liu, "Nanoscale ionic materials based on hydroxyl-functionalized graphene," Journal of Materials Chemistry A, vol. 2, no. 5, pp. 1409-1417, 2014.

[17] H. A. Barnes, "Shear-thickening ('dilatancy') in suspensions of nonaggregating solid particles dispersed in Newtonian liquids," Journal of Rheology, vol. 33, no. 2, pp. 329-366, 1989.

[18] E. Brown, N. A. Forman, C. S. Orellana et al., "Generality of shear thickening in dense suspensions," Nature Materials, vol. 9, no. 3, pp. 220-224, 2010.

[19] X. Cheng, J. H. McCoy, J. N. Israelachvili, and I. Cohen, "Imaging the microscopic structure of shear thinning and thickening colloidal suspensions," Science, vol. 333, no. 6047, pp. 1276-1279, 2011.

[20] M. I. Smith, R. Besseling, M. E. Cates, and V. Bertola, "Dilatancy in the flow and fracture of stretched colloidal suspensions," Nature Communications, vol. 1, no. 8, article 114, 2010.

[21] Y. Lee, E. D. Wetzel, and N. J. Wagner, "The ballistic impact characteristics of Kevlar woven fabrics impregnated with a colloidal shear thickening fluid," Journal of Materials Science, vol. 38, no. 13, pp. 2825-2833, 2003.

[22] R. L. Hoffman, "Explanations for the cause of shear thickening in concentrated colloidal suspensions," Journal of Rheology, vol. 42, no. 1, pp. 111-123, 1998.

[23] R. G. Egres and N. J. Wagner, "The rheology and microstructure of acicular precipitated calcium carbonate colloidal suspensions through the shear thickening transition," Journal of Rheology, vol. 49, no. 3, pp. 719-746, 2005.

[24] S. R. Waitukaitis and H. M. Jaeger, "Impact-activated solidification of dense suspensions via dynamic jamming fronts," Nature, vol. 487, no. 7406, pp. 205-209, 2012.

[25] G. Bossis and J. F. Brady, "The rheology of Brownian suspensions," The Journal of Chemical Physics, vol. 91, no. 3, pp. 1866$1874,1989$.

[26] J. F. Brady and G. Bossis, "The rheology of concentrated suspensions of spheres in simple shear flow by numerical simulation," Journal of Fluid Mechanics, vol. 155, pp. 105-129, 1985.

[27] N. J. Wagner and J. F. Brady, "Shear thickening in colloidal dispersions," Physics Today, vol. 62, no. 10, pp. 27-32, 2009.

[28] S. R. Raghavan, H. J. Walls, and S. A. Khan, "Rheology of silica dispersions in organic liquids: new evidence for solvation forces dictated by hydrogen bonding," Langmuir, vol. 16, no. 21, pp. 7920-7930, 2000.

[29] D. Li, M. B. Müller, S. Gilje, R. B. Kaner, and G. G. Wallace, "Processable aqueous dispersions of graphene nanosheets," Nature Nanotechnology, vol. 3, no. 2, pp. 101-105, 2008.

[30] R. L. Hoffman, "Discontinuous and dilatant viscosity behavior in concentrated suspensions. I. Observation of a flow instability," Transactions of the Society of Rheology, vol. 16, pp. 155-173, 1972.
[31] E. Brown and H. M. Jaeger, "The role of dilation and confining stresses in shear thickening of dense suspensions," Journal of Rheology, vol. 56, no. 4, pp. 875-923, 2012.

[32] Y. S. Lee and N. J. Wagner, "Dynamic properties of shear thickening colloidal suspensions," Rheologica Acta, vol. 42, no. 3, pp. 199-208, 2003.

[33] D. V. Kosynkin, G. Ceriotti, K. C. Wilson et al., "Graphene oxide as a high-performance fluid-loss-control additive in waterbased drilling fluids," ACS Applied Materials \& Interfaces, vol. 4, no. 1, pp. 222-227, 2012.

[34] W. Tesfai, P. Singh, Y. Shatilla, M. Iqbal, and A. Abdala, "Rheology and microstructure of dilute graphene oxide suspension," Journal of Nanoparticle Research, vol. 15, no. 10, article 1989, 2013.

[35] V. C. Tung, J. Kim, L. J. Cote, and J. Huang, "Sticky interconnect for solution-processed tandem solar cells," Journal of the American Chemical Society, vol. 133, no. 24, pp. 9262-9265, 2011. 

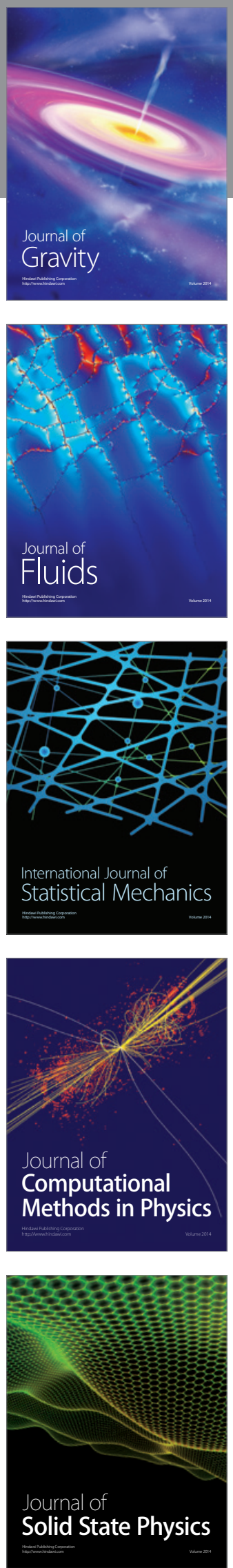

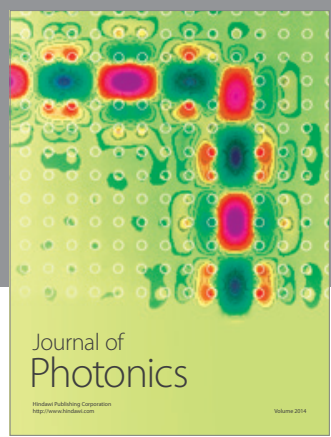

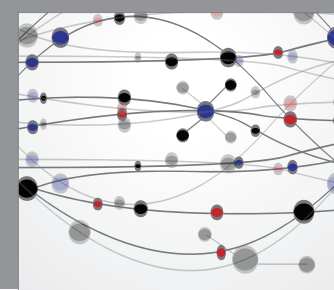

The Scientific World Journal

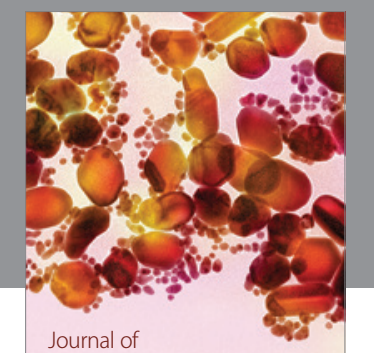

Soft Matter
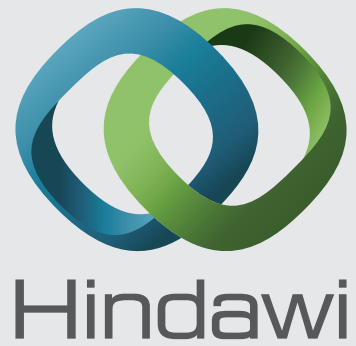

Submit your manuscripts at

http://www.hindawi.com
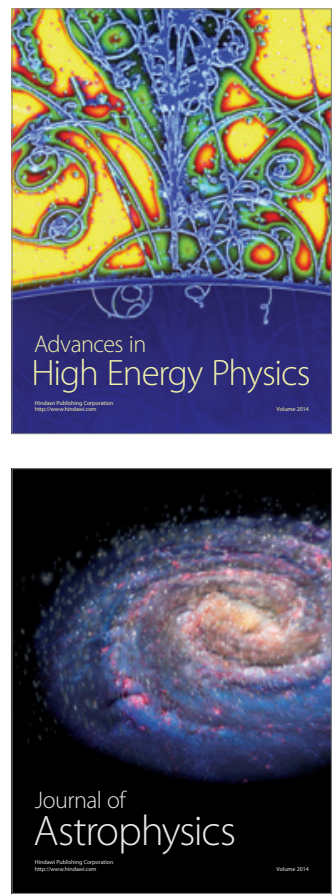
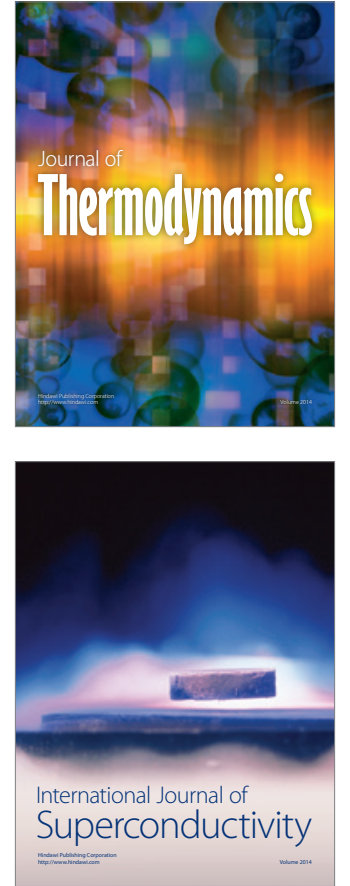
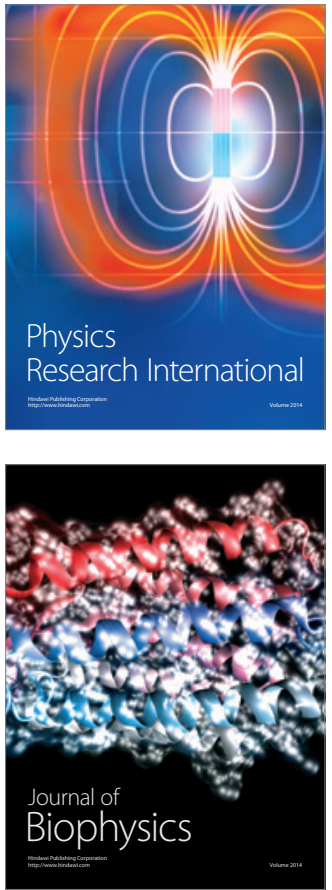
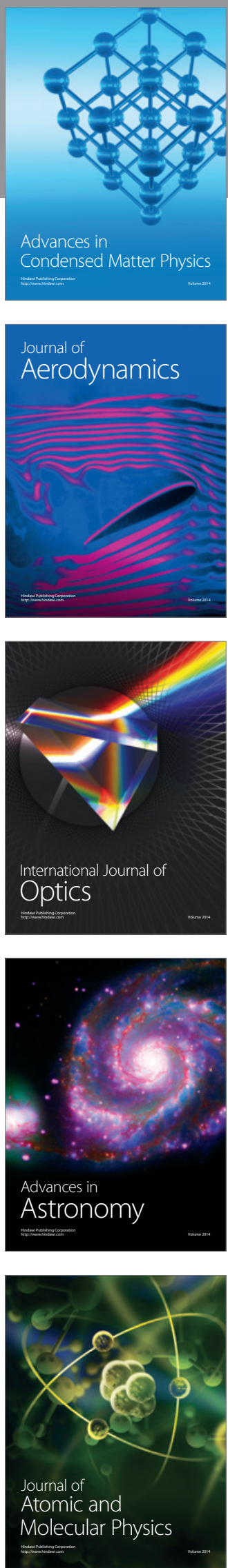